\title{
Video Article \\ Imaging Leukocyte Adhesion to the Vascular Endothelium at High Intraluminal Pressure
}

\author{
Danielle L. Michell ${ }^{1}$, Karen L. Andrews ${ }^{1}$, Kevin J. Woollard ${ }^{1}$, Jaye P.F. Chin-Dusting ${ }^{1}$ \\ ${ }^{1}$ Vascular Pharmacology Laboratory, Baker IDI Heart and Diabetes Institute, Monash University \\ Correspondence to: Jaye P.F. Chin-Dusting at jaye.chin-dusting@bakeridi.edu.au
}

URL: https://www.jove.com/video/3221

DOI: doi:10.3791/3221

Keywords: Immunology, Issue 54, Leukocyte adhesion, intraluminal pressure, endothelial dysfunction, inflammation, hypertension

Date Published: 8/23/2011

Citation: Michell, D.L., Andrews, K.L., Woollard, K.J., Chin-Dusting, J.P. Imaging Leukocyte Adhesion to the Vascular Endothelium at High Intraluminal Pressure. J. Vis. Exp. (54), e3221, doi:10.3791/3221 (2011).

\section{Abstract}

Worldwide, hypertension is reported to be in approximately a quarter of the population and is the leading biomedical risk factor for mortality worldwide. In the vasculature hypertension is associated with endothelial dysfunction and increased inflammation leading to atherosclerosis and various disease states such as chronic kidney disease ${ }^{2}$, stroke ${ }^{3}$ and heart failure ${ }^{4}$. An initial step in vascular inflammation leading to atherogenesis is the adhesion cascade which involves the rolling, tethering, adherence and subsequent transmigration of leukocytes through the endothelium. Recruitment and accumulation of leukocytes to the endothelium is mediated by an upregulation of adhesion molecules such as vascular cell adhesion molecule-1 (VCAM-1), intracellular cell adhesion molecule-1 (ICAM-1) and E-selectin as well as increases in cytokine and chemokine release and an upregulation of reactive oxygen species ${ }^{5}$. In vitro methods such as static adhesion assays help to determine mechanisms involved in cell-to-cell adhesion as well as the analysis of cell adhesion molecules. Methods employed in previous in vitro studies have demonstrated that acute increases in pressure on the endothelium can lead to monocyte adhesion, an upregulation of adhesion molecules and inflammatory markers ${ }^{6}$ however, similar to many in vitro assays, these findings have not been performed in real time under physiological flow conditions, nor with whole blood. Therefore, in vivo assays are increasingly utilised in animal models to demonstrate vascular inflammation and plaque development. Intravital microscopy is now widely used to assess leukocyte adhesion, rolling, migration and transmigration $^{7-9}$. When combining the effects of pressure on leukocyte to endothelial adhesion the in vivo studies are less extensive. One such study examines the real time effects of flow and shear on arterial growth and remodelling but inflammatory markers were only assessed via immunohistochemistry ${ }^{10}$. Here we present a model for recording leukocyte adhesion in real time in intact pressurised blood vessels using whole blood perfusion. The methodology is a modification of an ex vivo vessel chamber perfusion model ${ }^{9}$ which enables real-time analysis of leukocyte -endothelial adhesive interactions in intact vessels. Our modification enables the manipulation of the intraluminal pressure up to 200 $\mathrm{mmHg}$ allowing for study not only under physiological flow conditions but also pressure conditions. While pressure myography systems have been previously demonstrated to observe vessel wall and lumen diameter ${ }^{11}$ as well as vessel contraction this is the first time demonstrating leukocyte-endothelial interactions in real time. Here we demonstrate the technique using carotid arteries harvested from rats and cannulated to a custom-made flow chamber coupled to a fluorescent microscope. The vessel chamber is equipped with a large bottom coverglass allowing a large diameter objective lens with short working distance to image the vessel. Furthermore, selected agonist and/or antagonists can be utilized to further investigate the mechanisms controlling cell adhesion. Advantages of this method over intravital microscopy include no involvement of invasive surgery and therefore a higher throughput can be obtained. This method also enables the use of localised inhibitor treatment to the desired vessel whereas intravital only enables systemic inhibitor treatment.

\section{Video Link}

The video component of this article can be found at https://www.jove.com/video/3221/

\section{Protocol}

\section{Isolating carotid arteries}

1. Euthanase 10 week old Sprague Dawley rats via $\mathrm{CO}_{2} / \mathrm{O}_{2}$ asphyxiation

2. Excise left and right common carotid arteries with aorta and heart ensuring minimal stretching of the vessels.

3. In ice cold Krebs buffer separate the carotid arteries from the aorta and heart and perform close dissection.

4. Keep isolated vessels in Krebs on ice before mounting.

Approx. time $=45$ mins

\section{Priming the vessel chamber}

1. At the proximal and distal connectors of the vessel chamber flush Krebs buffer maintained at physiological pH by infusing carbogen gas (95\% $\mathrm{O}_{2} ; 5 \% \mathrm{CO}_{2}$ ) through the buffer at $37^{\circ} \mathrm{C}$. 
2. Ensure tubing and cannulas are flushed completely and are aligned.

3. Flush Kreb buffer through the P1 (proximal) and P2 (distal) transducers. Close taps to ensure no air bubbles in the transducers.

4. Connect the transducers to the corresponding connectors and again flush more Krebs buffer ensuring no air bubbles. Close off taps to the chamber.

Approx. time $=15$ mins

\section{Pressurizing the vessel chamber}

1. Turn on pressure equipment: pressure servo, pressure monitor, peristaltic pump (Figure 1).

2. With the peristaltic pump on pressure and the pressure servo on automatic run Krebs buffer through the tubing at $20 \mathrm{mmHg}$ (dial at 1 ), ensuring no air bubbles.

3. Connect tubing to the closed P2 transducer. Pressure will become stable.

4. Open P2 tap to the vessel chamber to flush out any bubbles then close off.

5. Fill the bath with Krebs buffer $(5-7 \mathrm{~mL})$.

Approx. time $=10$ mins

\section{Mounting the vessel}

1. Under a dissecting microscope place black polyester ties onto each cannula (Figure 2).

2. Move cannulas and cannula holders apart and mount $1 / 4$ vessel (aortic arch end) onto the $P 1$ cannula. Ensure not to tear the vessel.

3. Using a syringe filled with Krebs buffer gently flush excess blood out of the vessel via P1 transducer. Close off P1 to chamber.

4. Secure vessel to the P1 cannula with the polyester tie.

5. Move cannulas/cannula holders closer for mounting onto the P2 cannula.

6. Mount distal end of the vessel ( $1 / 4$ of the length) onto distal cannula. Secure with polyester tie.

Approx. time $=20$ mins

\section{Pressurizing the vessel}

1. Adjust the cannula holders to ensure no bend or stretch in the vessel.

2. With $\mathrm{P} 1$ and $\mathrm{P} 2$ closed to the chamber switch the pressure servo from automatic to manual. Check on the pressure servo there is no continual decrease in pressure within 10 seconds. If there is, a leak is occurring and the connections and transducers need to be secured in place.

3. Adjust the pressure servo back to automatic then open P2 to the chamber. Under the microscope observe the vessel dilate when the tap is opened to the chamber.

4. Switch the pressure servo to manual. Again check for continual decrease in pressure within 10 seconds. If there is a leak then the system is not pressure tight and there is likely to be a hole in the vessel.

5. Switch back to automatic open $\mathrm{P} 1$ to the chamber. On the manual setting check that the pressure remains stable.

6. If no leak, on manual setting increase dial to $2(40 \mathrm{mmHg})$.

7. Adjust to automatic and observe the vessel under the microscope. If necessary, adjust the cannula holder to ensure no bend in the vessel.Check for leaks on manual setting.

8. Repeat steps $5.6-5.7$ increasing the dial to $3(60 \mathrm{mmHg}), 4(80 \mathrm{mmHg})$ and so on until desired pressure is reached.

Approx. time $=15-30$ mins

\section{Incubating the pressurized vessel}

1. Connect the temperature controller set to $37^{\circ} \mathrm{C}$.

2. With a second peristaltic pump perfuse Krebs buffer $(1 \mathrm{~mL} / \mathrm{min})$ into the vessel chamber bath.

3. Connect aspirator to chamber ensuring only the top layer of the bath is removed.

4. Incubate pressurized vessel at $37^{\circ} \mathrm{C}$ for 1 hour with the pressure set to automatic.

5. Check the pressure is not leaking (switching to manual) periodically.

6. The effects of various pharmacological interventions can be observed by simply adding the compound to the bath during incubation.

Approx. time $=1$ hour

\section{Perfusing the pressurized vessel with whole blood}

1. During incubation obtain at least $7.5 \mathrm{~mL}$ human whole blood $/ \mathrm{vessel}$ collected in $40 \mathrm{U}$ heparin $/ \mathrm{mL}$ blood. Incubate in a $50 \mathrm{ml}$ falcon at $37^{\circ} \mathrm{C}$.

2. 10 minutes before the end of the incubation label blood with VybrantDil $(1: 1000)$ for 10 minutes at $37^{\circ} \mathrm{C}$ in dark.

3. After 10 minutes, collect blood in a syringe clearing any bubbles and attach onto a syringe pump with a heat jacket set at $37^{\circ} \mathrm{C}$.

4. Close off $\mathrm{P} 1$ transducer to the chamber/vessel and connect syringe and waste tubing.

5. Purge blood at $1000 \mu \mathrm{L} / \mathrm{min}$ through the waste tubing to remove any bubbles.

6. Open $\mathrm{P} 1$ to the chamber. The pressure servo will adjust automatically to maintain desired pressure.

7. Perfuse blood at $100 \mu \mathrm{L} / \mathrm{min}$. 
8. Using a fluorescent microscope coupled to a digital camera record two fields of the perfused vessel for 15 seconds at $1,3,5,7.5$ and 10 minutes.

9. Post perfusion endothelial integrity and function can be assessed via pharmacological techniques such as myograph and adhesion molecule expression can be determined by immunohistochemistry to further validate the inflammatory response.

Approx. time $=15$ mins

\section{Representative Results:}

A schematic diagram of the pressure chamber setup is shown in Figure 1. With a digital camera coupled to a fluorescent microscope results can be visualised instantly via live video recordings. Representative video images are seen in Figure $\mathbf{3}$ where leukocytes are considered to be adherent to the endothelium if they remained stationary for 10 seconds. With video recordings on continuous loop adhered cells can be counted as an average per field. While both low (Figure 3A) and high (Figure 3B) pressure will cause some amount of adhesion a significant increase in leukocyte adhesion at the higher intraluminal pressure is seen and this is also demonstrated quantitatively (Figure 4).

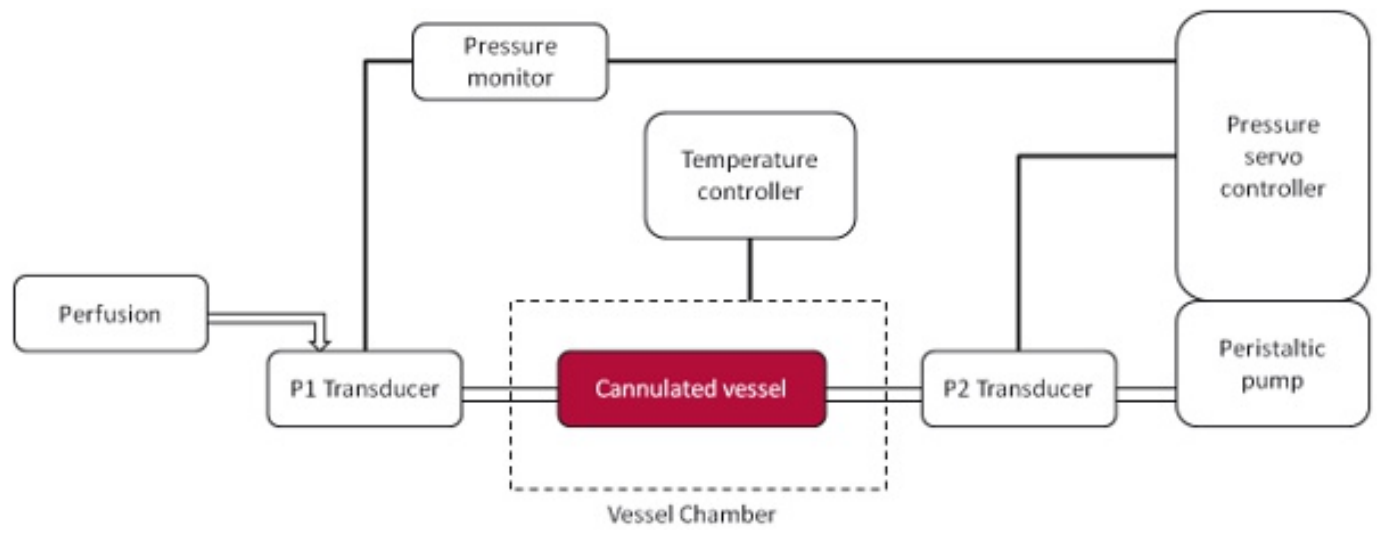

Figure 1. Pressurised ex vivo vessel chamber schematic. A cannulated vessel connected to a proximal (P1) transducer and a distal (P2) transducers that enables blood pressure to be manipulated within the vessel. Perfusion is through the P1 transducer and pressure is maintained via P2 transducer.

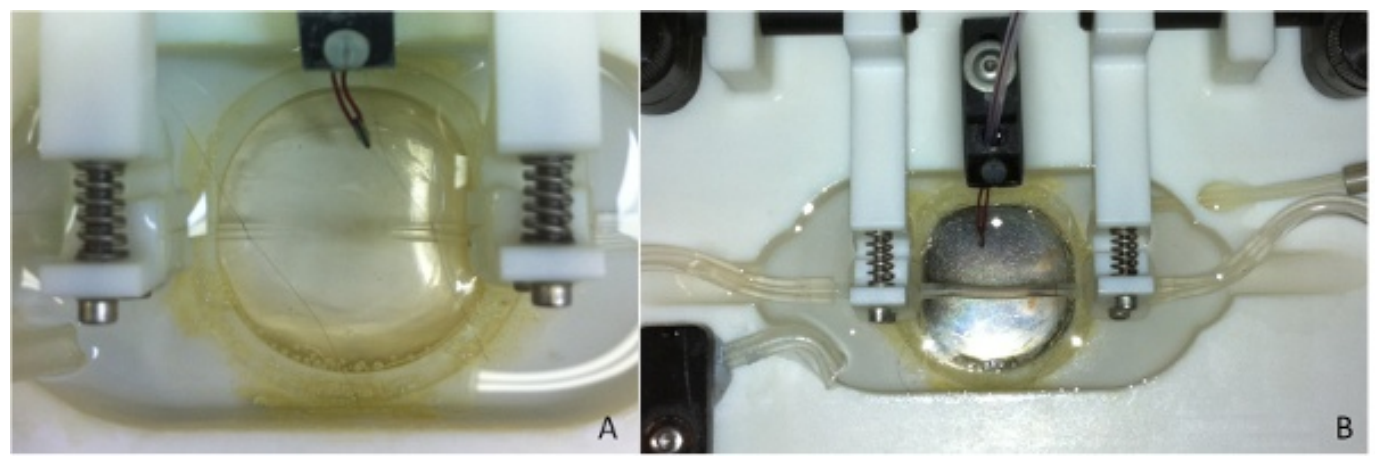

Figure 2. Cannuala with ties. Black polyester ties are attached to each cannula. 

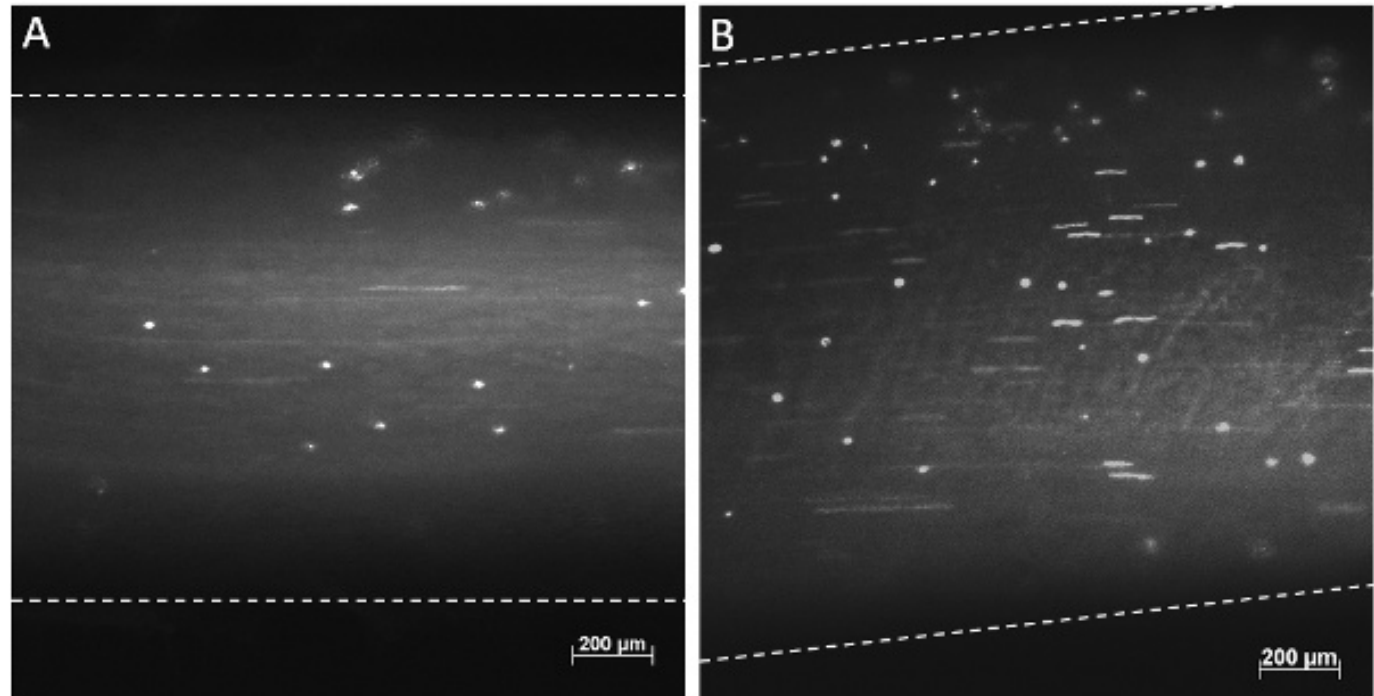

Figure 3. Representative video images. Dynamic cell adhesion (red arrow) under fluorescence at $80 \mathrm{mmHg}(\mathrm{A})$ and $120 \mathrm{mmHg}(\mathrm{B})$ after 10 minutes of perfusion.

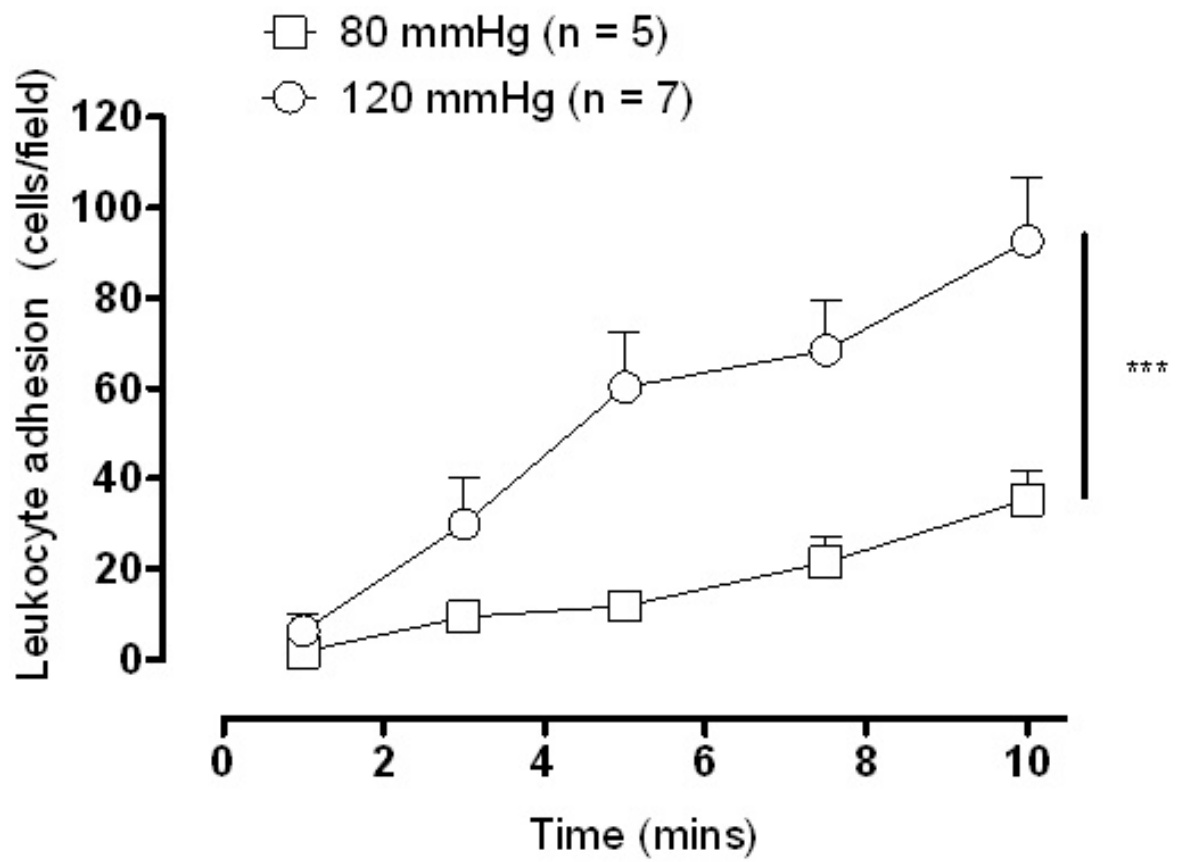

Figure 4. Leukocyte adhesion in Sprague Dawley carotid arteries after 1 hour incubation at low (80 $\mathrm{mmHg})$ and high pressure (120 mmHg). ${ }^{* * *} \mathrm{P}<0.001$, as analysed by 2-way repeated measures ANOVA using Bonferroni post hoc test.

\section{Discussion}

This is a modified method to study leukocyte adhesion to the endothelium in intact isolated blood vessels under pressurised conditions in real time. Perfusion of the vessel chamber alone enables a quick validation of pro-inflammatory strains of large mice and rat vessels. Enabling pressure manipulation allows dynamic cell interactions to be observed from low to very high intraluminal pressures, thus better mimic-ing physiological and pathophysiological conditions. Diameter of vessels can also be measured using a sufficient cell-imaging program and therefore shear flow and rate can be determined and therefore manipulated.

With its myograph capabilities, pharmacological interventions placed in the bath add another dimension to the experimental conditions possible with this model enabling studies of mechanistic and signalling pathways. While endothelial preservation cannot be confirmed during pressure manipulation, responses to ACh and PE can be conducted post perfusion ${ }^{9}$.

It should be noted that this setup demonstrates the effects of intraluminal pressure on cell-to-cell interactions not the effects of pulsatile blood flow nor systolic or diastolic pressures. Furthermore, while acute pressure changes on leukocyte adhesion were observed, this setup can be also utilised to look at chronic pressure effects (ie increasing incubation times and using a chronic pressure animal model). Sprague Dawley common carotid arteries are demonstrated in this set up but other strains and species may be used with appropriate adjustments to the cannula size. 
Indeed, it is important to note that the age and weight of animals affect vessel size and thus that the set up needs to be individualised for each vessel. Close and careful dissection of the connective tissue can improve visualisation of leukocytes immensely.

\section{Disclosures}

The study protocol was approved by the Alfred Medical Research and Education Precinct Animal Ethics Committee and the Alfred Hospital Ethics Committee.

\section{Acknowledgements}

This study was supported in part by the Victorian Government's OIS Program, the National Health and Medical Research Council of Australia program and project grants (JPF Chin-Dusting) and postgraduate scholarship (D Michell).

\section{References}

1. Lopez, A.D., Mathers, C.D., Ezzati, M., Jamison, D.T., \& Murray, C.J. Global and regional burden of disease and risk factors, 2001: systematic analysis of population health data. Lancet. 367 (9524), 1747-1757 (2006).

2. Szczech, L.A. et al. Acute kidney injury and cardiovascular outcomes in acute severe hypertension. Circulation. 121 (20), $2183-2191$.

3. Rodriguez-Garcia, J.L., Botia, E., de La Sierra, A., Villanueva, M.A., \& Gonzalez-Spinola, J. Significance of elevated blood pressure and its management on the short-term outcome of patients with acute ischemic stroke. Am J Hypertens. 18 (3), $379-384$ (2005).

4. Levy, D., Larson, M.G., Vasan, R.S., Kannel, W.B., \& Ho, K.K. The progression from hypertension to congestive heart failure. JAMA. 275 (20), 1557-1562 (1996).

5. Ley, K., Laudanna, C., Cybulsky, M.I., \& Nourshargh, S. Getting to the site of inflammation: the leukocyte adhesion cascade updated. Nat Rev Immunol. 7 (9), 678-689 (2007).

6. Riou, S. et al. High pressure promotes monocyte adhesion to the vascular wall. Circ Res. 100 (8), 1226-1233 (2007).

7. Ley, K. \& Gaehtgens, P. Endothelial, not hemodynamic, differences are responsible for preferential leukocyte rolling in rat mesenteric venules. Circ Res. 69 (4), 1034-1041 (1991).

8. Bernhagen, J. et al. MIF is a noncognate ligand of CXC chemokine receptors in inflammatory and atherogenic cell recruitment. Nat Med. 13 (5), 587-596 (2007).

9. Woollard, K.J. et al. Pathophysiological levels of soluble P-selectin mediate adhesion of leukocytes to the endothelium through Mac-1 activation. Circ Res. 103 (10), 1128-1138 (2008).

10. Eberth, J.F. et al. Importance of pulsatility in hypertensive carotid artery growth and remodeling. J Hypertens. 27 (10), $2010-2021$ (2009).

11. Cooke, J.P., Rossitch, E., Jr., Andon, N.A., Loscalzo, J., \& Dzau, V.J. Flow activates an endothelial potassium channel to release an endogenous nitrovasodilator. J Clin Invest. 88 (5), 1663-1671 (1991). 